\title{
A Functional MRI Index of Spatial Context Effects in Vision
}

\author{
Keith A. Feigenson 1,2*, Catherine Hanson ${ }^{3}$, Thomas Papathomas', Steven M. Silverstein 1,5 \\ ${ }^{1}$ Department of Psychiatry, Robert Wood Johnson Medical School at Rutgers, The State University of New \\ Jersey, Piscataway, USA \\ ${ }^{2}$ Albright College, Reading, PA, USA \\ ${ }^{3}$ Rutgers University Brain Imaging Center, Newark, NJ, USA \\ ${ }^{4}$ Department of Biomedical Engineering and Center for Cognitive Science, Rutgers University, Piscataway, NJ, \\ USA \\ ${ }^{5}$ University Behavioral Health Care at Rutgers, The State University of New Jersey, Piscataway, NJ, USA \\ Email: "kfeigenson@albright.edu
}

Received 25 October 2015; accepted 28 December 2015; published 31 December 2015

Copyright (C) 2015 by authors and Scientific Research Publishing Inc.

This work is licensed under the Creative Commons Attribution International License (CC BY).

http://creativecommons.org/licenses/by/4.0/

(c) (i) Open Access

\begin{abstract}
Coordination of brain activity, in the form of modulation of feedforward activity by stored information and expectations, occurs across domains of perception and cognition. A reliable and compelling example of this is size contrast in vision. This paper builds on a prior study to show that in healthy humans, the spread of activation in striate and extrastriate visual cortex during a context-modulated size perception task is dependent on the perceived size of the target image, not on the physical size of the retinal image. These data provide further evidence that early regions in visual cortex are modulated by top-down influences, and provide a framework for investigating visual context processing in psychiatric disorders where reduced sensitivity to visual contextual effects has been demonstrated in behavioral tasks.
\end{abstract}

\section{Keywords}

fMRI, Size Perception, Contrast, Visual Illusion, Mental Health

\section{Introduction}

While neuroscience has made significant advances towards understanding the functions and firing properties of individual neurons and brain regions, the topic of their coordinated activity has received far less attention. Nev-

${ }^{*}$ Corresponding author. 
ertheless, dynamic coordination of neural activity (i.e., modulation of signals based on current context) is essential for successful adaptation (Phillips, Clark, \& Silverstein, 2015). This concept is now well established in vision, where the influence of visual stimuli outside of the classical receptive field on primary visual cortex neurons has been convincingly demonstrated (Freeman, Sagi, \& Driver, 2001; Zhang \& Von der Heydt, 2010; Zhu \& Rozell, 2013). This evidence has transformed the traditional view that V1 provides only a topographical representation of retinotopic information (Tootell et al., 1998). Less work has been done on the neural effects of higher cognitive influences on perception, although many behavioral studies have demonstrated the effects of emotional state (Damaraju, Huang, Barrett, \& Pessoa, 2009; Hu, Padmala, \& Pessoa, 2013; Keil et al., 2010), focus of attention (Fang, Boyaci, Kersten, \& Murray, 2008; Gilbert, Ito, Kapadia, \& Westheimer, 2000), stored information (learning and memory) (Doherty, Campbell, Tsuji, \& Phillips, 2010; Doherty, Tsuji, \& Phillips, 2008; Li, Piech, \& Gilbert, 2008; Papathomas \& Bono, 2004), expectations (Silverstein et al., 2006; Silverstein \& Keane, 2009; Silverstein et al., 1996; Vetter \& Newen, 2014), and social context (Kret \& de Gelder, 2010) on visual processing.

In an fMRI study using a size illusion task, Murray and colleagues demonstrated that the spatial distribution of activity in V1 reflected the perceived size of an image, rather than the retinal size (Murray, Boyaci, \& Kersten, 2006). More recently, similar findings have been reported using variants of the Ebbinghaus and Ponzo illusions by Fang et al. (2008), Schwarzkopf et al. (2011), and Schwarzkopf \& Rees (2013). In the present study, we aimed to confirm this basic effect and further explore contextual effects in visual cortex. We replicated the timing and duration of the trial presentation used by Murray et al. (2006). However, to more closely assay realworld experience, we presented realistic photographic images rather than the drawn geometric shapes used in that study. The aforementioned studies used target stimuli of basic geometric shapes to maximally excite neurons in V1; these were not shapes that individuals would typically observe outside of the laboratory [we note that while Schwarzkopf et al. (2011) used a photograph of a tunnel for background stimuli, the targets were simple black and white circle sketches]. While simple object processing-related activity in early visual cortex can inform our understanding of more abstract and complex object processing (for review, see Wilson \& Wilkinson, 2015), natural image viewing activates additional brain regions. For example, categorical image processing activates inferior temporal cortex (Carlson, Ritchie, Kriegeskorte, Durvasula, \& Ma, 2014; Ritchie, Tovar, \& Carlson, 2015) and object recognition activates multiple points along the ventral processing stream and into higher cortical areas (Reddy \& Kanwisher, 2006). This difference may be partly explained by additional feedback interactions involved in natural object processing, relative to simple shape processing.

In the present study, identically sized target photographic images were presented in spatial contexts such that they were judged to be closer to, or further from, the observer. The dependent variable was spread of activity in V1 - V5, and whether this was related to perceived image size.

\section{Materials and Methods}

\subsection{Subjects}

Subjects were recruited from the Rutgers-Newark Psychology Department subject pool and received course credit for participation (subject demographics are shown in Table 1). Eighteen subjects (12 female) were initially recruited. All subjects signed a consent form and the study protocol was reviewed and approved by the Rutgers University Institutional Review Board. All participants were between the ages of 18 - 56, and, based on a set of standard questions, denied having a history of psychotic or neurologic illness, serious head injury, or drug use in the prior 6 months. One subject was unable to participate in the scanning session, and so was removed from analysis. There was an anomaly detected in the brain scan of another subject, who was also removed from analysis, yielding a final analysis sample of 16 (10 female). All subjects were characterized by normal levels of intelligence, as determined by the Shipley-2 Institute of Living Scale vocabulary and abstraction components (Shipley, Gruber, Martin, \& Klein, 2009).

\subsection{Stimuli and Design}

Figure 1 displays the four stimuli that were used to induce a size illusion effect in this study. Two of the stimuli depicted a white cat in a tunnel, one at the front of the tunnel (i.e., "near") and the other at the back of the tunnel (i.e., "far"). The other two images depicted a white dog of similar size at the front or the back of the tunnel. The 
Table 1. Demographic information of study participants.

\begin{tabular}{cc}
\hline Total Participants & 16 \\
Age (Years) & $21.3 \pm 3.4$ \\
Sex (\# Female) & 10 \\
Race (\#) & 0 \\
White & 1 \\
Black & 6 \\
Hispanic & 6 \\
Asian & 3 \\
Other & $14.1 \pm 2.9$ \\
Education (Years) & $100.2 \pm 10.0$ \\
Shipley-2 Score & \\
\hline
\end{tabular}
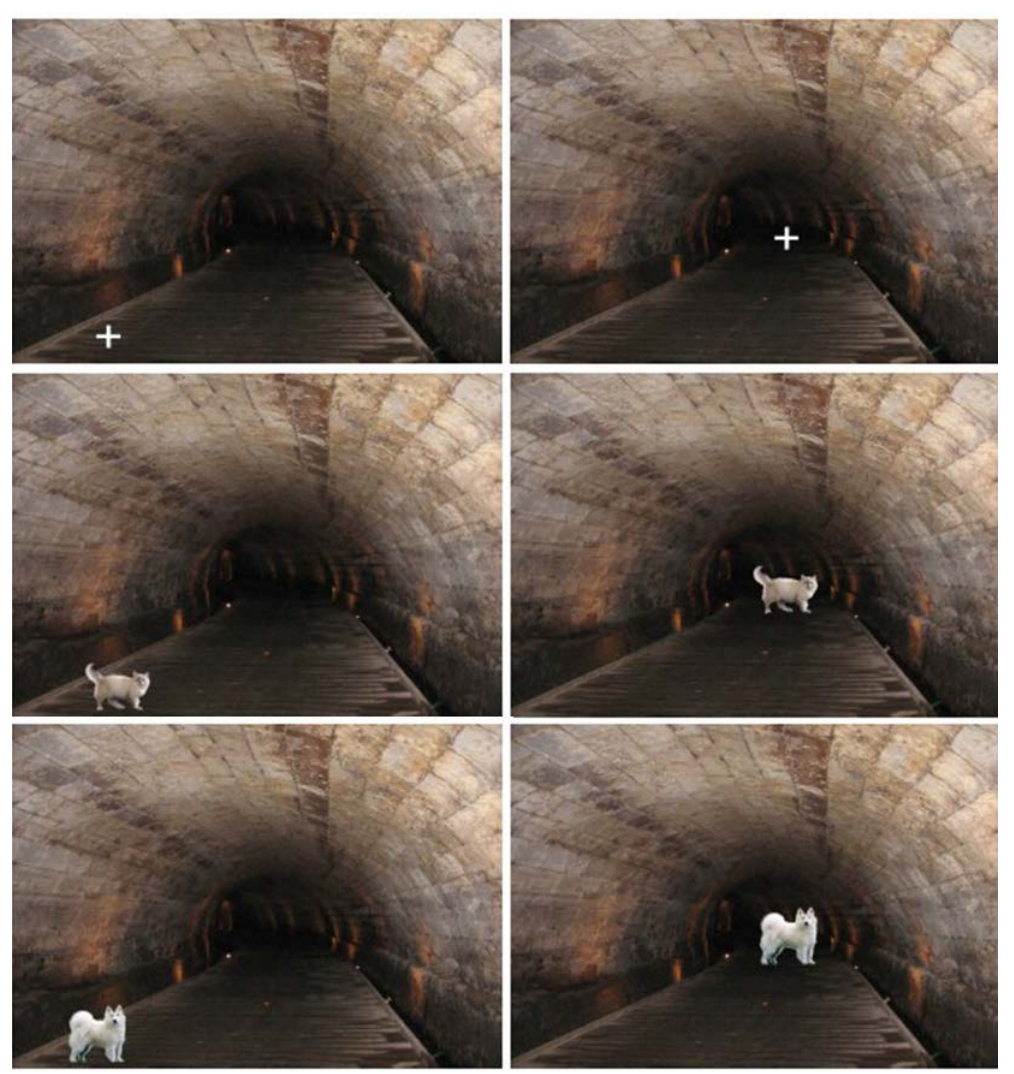

Figure 1. Stimuli used for imaging. The textured background was universally present. Subjects viewed static images for 10 seconds. At first, a fixation image would appear, on which subjects were to focus with the knowledge that would be where the next target image would appear. This could be presented in the form of a dog or a cat. Images were randomly presented as either cats or dogs in either the center of the background ("far" condition) or the bottom left ("near condition"). Each condition was presented an equal number of times. The degrees of visual angle subtended by each cat image were $3.9^{\circ} \times 3.1^{\circ}$. The degrees of visual angle subtended by each dog image were $3.7^{\circ} \times 3.5^{\circ}$. The standard background image subtended $29.4^{\circ} \times 22.3^{\circ}$. 
tunnel background was identical for all four images. The viewing distance, including distance of eyes to the mirror, was $990 \mathrm{~mm}$.

Stimulus presentation and response collection were done using PsychoPy (Peirce, 2007, 2008). The images were presented serially, each one for 10 seconds. Prior to a stimulus being presented, a white fixation cross was shown for 10 seconds in the position that the dog or cat would appear, so the duration of any given trial (fixation and stimulus) was 20 seconds, as in the study by Murray et al. (2006). To assure that subjects attended to the stimuli, they were asked to press a response button when they saw a "cat" and another button when they saw a "dog". Figure 2 displays an example of two trial presentations. Each condition was presented 5 times in a sequence randomized for each subject, and was scored as correct or incorrect.

Just prior to the scanning session, and before entering the scanner, a behavioral measure of the size illusion effect was obtained for each subject. The task consisted of the same "target" cat and dog stimuli presented against the tunnel background. However, for each trial, a "probe" cat or dog (i.e., the same stimuli used in the fMRI experiment) was presented at the right of the stimulus image, in the absence of context. Subjects used the arrow keys to adjust the size of the probe cat or dog until they believed it was the same size as the target object, at which point they pressed the spacebar for the next stimulus. Subjects were not timed for this task. Each condition was presented 8 times in a sequence randomized for each subject, and for each trial, the probe (cat or dog) to be manipulated was randomly scaled, such that it was either larger or smaller than the target object, but the average ratio of probe to target was 1.0. For analysis, we used the variable of final ratio of probe object size relative to the target object size.

\section{3. fMRI Protocol}

Subjects viewed the stimuli and made responses while being scanned in a Siemens 3T Trio magnet. Subjects were scanned in a prone position and a 12-channel Siemens head coil was used. Foam cushioning was used to stabilize head position and minimize head movement. An MRI-compatible button box was used to record responses. The stimuli were back projected onto a screen covering the rear bore of the magnet. A mirror attached to the head coil allowed subjects to view the stimuli in the normal orientation. The presentation of stimuli and

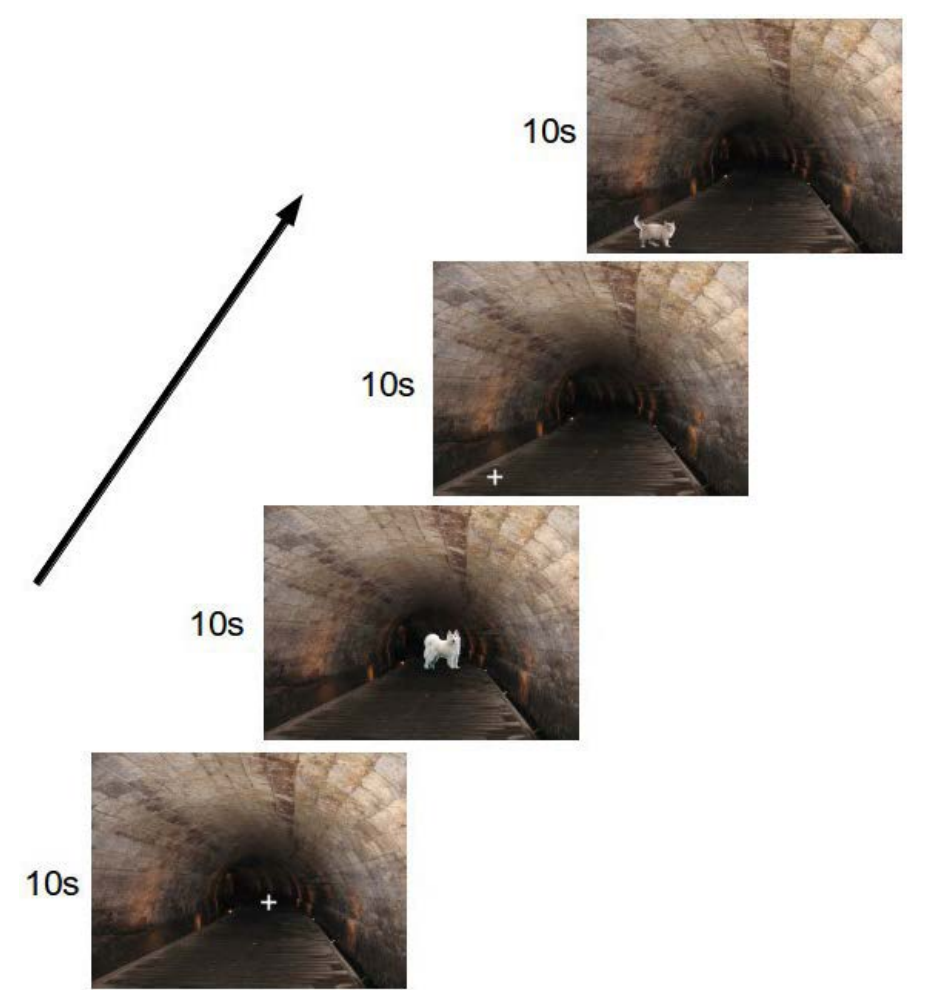

Figure 2. Example of stimuli presentation sequence. Each separate image (target or fixation) was presented for 10 seconds. 
recording of responses was done using PsychoPy. Scanning was synchronized with stimulus presentation through a trigger pulse sent to the PsychoPy software.

Prior to the experimental trial sequence, a T1-weighted axial anatomical scan $(\mathrm{TR}=2000 \mathrm{~ms}$, $\mathrm{TE}=4.38 \mathrm{~ms}$, $204 \times 256$ matrix, FOV $=22 \mathrm{~cm}$, slice thickness $2 \mathrm{~mm}, 0 \mathrm{~mm}$ gap, 80 slices) was obtained for each subject and used to register the functional imaging data for that subject during analysis. Functional imaging was done using an echo planar gradient echo imaging sequence and axial orientation, and data were obtained using the following parameters: TR $=2000 \mathrm{~ms}$, TE $=30 \mathrm{~ms}, 64 \times 64$ matrix, FOV $=22 \mathrm{~cm}$, slice thickness $4 \mathrm{~mm}, 0 \mathrm{~mm}$ gap, 32 slices.

Preparation of the BOLD data and statistical analyses were performed using FSL (FMRIB's Software Library, http://www.fmrib.ox.ac.uk/fsl). BOLD data from each subject was skull-stripped using BET, FSL's brain extraction tool (Smith, 2002) and then registered first to that subject's anatomical data and then to the $2 \mathrm{~mm}$ MNI template space using FLIRT, FSL's registration tool (Jenkinson, Bannister, Brady, \& Smith, 2002). A General Linear Model (GLM) analysis using FSL's modelling tool, FEAT (Woolrich, Ripley, Brady, \& Smith, 2001) was performed first for each subject, and then across subjects in a second analysis.

For each analysis of an individual subject's data, the skull-stripped BOLD data for that subject was motion corrected (FSL's McFlirt tool) and prewhitened (FSL's FILM). A high-pass filter (100 seconds) and 5 mm spatial smoothing were applied. For the group analysis, a mask comprised of visual areas V1 through V5 was constructed using the Juelich histological atlas, and the GLM analysis was restricted to voxels included in that mask. A mixed-effects model (FSL's FLAME) was applied using a corrected cluster threshold of $p=.001$. Group-level analyses were based on four contrasts used during the subject-level analyses: far (cat + dog), near (cat $+\operatorname{dog})$, far $>$ near, and near $>$ far.

\section{Results}

\subsection{Behavioral Measures}

To observe if our image manipulation was effective, we performed a $t$-test between the final adjusted ratio of manipulated probe image size to target image size when the target image was presented on the bottom left side of the screen (i.e., when it was perceived as "near") compared to when it was presented in the middle part of the screen (i.e., when it was perceived as "far"). Using a one sample $t$-test, with 1 as the reference point, we observed that subjects significantly over-adjusted the size of the "far" image by close to $5 \%[t(15)=4.611, p$ $<.001, \mathrm{M}=1.046, \mathrm{SD}=.04]$. In contrast, subjects were largely accurate when adjusting the object to match the "near" target $[t(15)=1.356, p=.195, \mathrm{M}=1.014, \mathrm{SD}=.04]$. When the average estimated size of the "far" object was compared to that of the "near" object by means of a paired $t$-test, the difference was also significant $[t(15)=$ $3.51, p<.01, \mathrm{M}=.032, \mathrm{SD}=.04]$. This difference remained significant whether the difference was analyzed by image type as dog $[t(15)=2.88, p<.05]$ or cat $[t(15)=2.74, p<.05]$. To obtain an overall measure of contextual influence, we subtracted the 'near' target ratio from the 'far' target ratio for each subject. This value was not correlated with age ( $r=-.354, p=.18$ ), years of education $(r=-.338, p=.22)$, or Shipley-Vocabulary score $(r=$ $-.413, p=.13)$. In addition, there were no differences on this score between males and females $[t(14)=.52, p$ $=.61]$ or racial groups $[F(2,12)=.353, p=.79]$.

\subsection{Imaging}

Subjects' proportion correct responses $(\mathrm{M}=.988, \mathrm{SD}=.029)$ indicated that all subjects were paying attention to the stimulus in the scanner. In order to assess the effect of the size illusion in visual cortex regions (V1 through V5), voxel activation generated from the "far" stimuli (cat and dog) was contrasted with that generated by the "near" (cat and dog). Figure 3 shows areas of significantly greater activation in response to the "far" stimuli compared to the "near" stimuli, within the V1 - V5 mask. Greater activity was observed in response to the "far" stimuli, primarily in V1 and V2 but also extending into higher visual regions. Note that this difference did not remain when we subtracted activation related to perception of the fixation point. This is because there was extreme overlap in visual cortex activation for the fixation point stimulus and the target stimuli. Therefore, subtracting activation due to viewing the fixation stimulus from activation due to viewing the target stimulus removed much of the data used in the original contrast (data not shown). Also, given that perception of the fixation point should be subject to the same illusory effects as perception of the target stimuli, removing variance related to fixation point perception would be expected to reduce or negate the "far-minus-near" effect. 

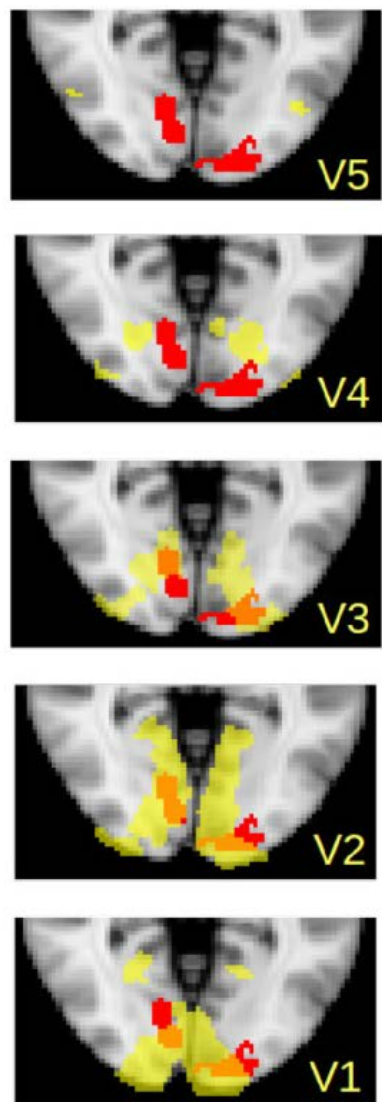

Figure 3. Cortex imaging. Images of occipital cortex showing the significant activation of voxels in the visual areas that responded to the "far" stimuli more than to the "near" stimuli. Differential activation was primarily observed in V1 and V2, but extended into higher visual areas (e.g., V3). The slice coordinates are: $x=45, y=13, z=35$. The mask is indicated by the yellow pixels, and the activation is indicated by the red pixels. All clusters were significant at $p$ $<.001$. There were 7 clusters with maxima at the following coordinates $(\mathrm{mm})$ : 1) $\mathrm{x}=1-10, \mathrm{y}=-100, \mathrm{z}=4$; 2) $\mathrm{x}=-4, \mathrm{y}=-94, \mathrm{z}=-14$; 3) $\mathrm{x}=-44, \mathrm{y}=-78$, $\mathrm{z}=2$; 4) $\mathrm{x}=6, \mathrm{y}=-76, \mathrm{z}=26$; 5) $\mathrm{x}=-18, \mathrm{y}=-98, \mathrm{z}=22$; 6) $\mathrm{x}=32, \mathrm{y}=-96$, $\mathrm{z}=12 ; 7) \mathrm{x}=-32, \mathrm{y}=-92, \mathrm{z}=-12$.

\section{Discussion}

We observed that equally sized images of real-world objects can differentially affect the extent and distribution of activation in early visual cortex — as a function of their spatial context—illustrated by the voxels activated in V1-V3 by the "far" target images but not the "near" target images. This is an extension of previous findings (Fang et al., 2008; Murray et al., 2006), showing for the first time that same-sized target stimuli not specifically created to maximize neural firing in V1 can nevertheless induce differential activity in primary visual cortex in relation to spatial and textural context. In the context of increasing awareness of the non-replicability of research findings (Open Science, 2015), we believe our study supports this brain basis for a basic visual function. This is especially relevant since it increases confidence in the potential utility of this measure for studies of clinical populations (see below).

Our study engaged participants in an active behavioral task while in the scanner, requiring participants to engage attentional resources to make a decision based on semantic categorization. This differs from previous studies involving contextual effects on visual perception that used passive participation as a means of eliminating higher-order functions (such as attention) from interacting with activation of basic visual cortex (Murray et al., 2006; Pooresmaeili, Arrighi, Biagi, \& Morrone, 2013). However, other studies using active participation have shown similar size constancy effects to that of Murray et al. (2006) (Fang et al., 2008; Schwarzkopf \& Rees, 
2013; Schwarzkopf, Song, \& Rees, 2011). Our study thus confirms that these effects in early visual cortex can be obtained during conditions of active attention.

Interestingly, in our behavioral task, we did not find that subjects underrepresented the size of the "near" object (in the bottom left); indeed, subjects were largely accurate in manipulating the size of the object when the target was closer. This could be due to the greater amount of context surrounding the "far" images (which appeared closer to the center of the screen, and were surrounded by context to an approximately equal degree on all sides) compared to the "near" images (which appeared close to the bottom, and so were less surrounded by context in the lower portion of the images). However, it is also possible that distortions of perceived visual angle are simply greater for objects that are perceived as being further from the observer (Arnold, Birt, \& Wallis, 2008). Consistent with this, Merriman et al. (2010) observed that adults use size estimation strategies at far, but not near, distances.

We believe that, for several reasons, our results could be used to guide investigations of the effects of regional brain volumes on perception. First, individual differences across a wide range of human behaviors correlate with individual differences in brain anatomy (for review, see Kanai \& Rees, 2011). Next, it has been shown that individual variability in surface area of $\mathrm{V} 1$ is a reliable indicator of the strength of size context illusions (Schwarzkopf et al., 2011), with larger surface area equating to decreased lateral inhibition between neurons (Kaas, 2000) and a subsequent decrease in contextual illusion strength. These effects may extend to other visual stimuli, including contrast, luminance, and orientation (Song, Schwarzkopf, \& Rees, 2013). Electrophysiological data complement these findings, showing that V1 cell receptive fields will change position depending on monocular depth cues (Ni, Murray, \& Horwitz, 2014). The extent of this change, however, is limited by physical anatomy, as described above. This literature collectively shows that visual context processing varies as a result of differences in extent of lateral inhibitory connections, and these variations can be reliably measured at anatomical and functional levels.

There are several notable limitations to this study. A difference between this study and others (Murray et al., 2006; Schwarzkopf et al., 2011) that examined contextual processing in early visual cortex is that we did not perform retinotopic mapping (i.e., specifically defining V1 in every subject), but rather, used the Juelich histological atlas to define visual regions of interest. As a result, it is possible that some of the activity we detected in extrastriate regions was due to imprecision in measurement of V1 in our grouped data. It must be noted, however, that the use of V1 localizers has been criticized (as being potentially biased and even unnecessary), especially in cases where significant feedback from higher-level cognition to V1 is expected (Friston, Rotshtein, Geng, Sterzer, \& Henson, 2006). In addition, because extrastriate regions process progressively larger areas of space with increasing distance from V1 (i.e., V2, V3, V4, etc.) (Angelucci et al., 2002), the effects we observed in extrastriate regions may reflect real differences in perceived stimulus size, information about which is fed back to V1, rather than being due to anatomical overlap of occipital regions across subjects. Murray et al. (2006) only examined ROIs within V1 and so did not present data on this issue, although they did recognize that some of their V1 effects could be due to feedback from higher-order visual regions. A third study limitation is that the number of trials per stimulus condition were limited, and although the design had been used before (Murray et al., 2006; Song et al., 2013), additional trials for each of our conditions may have allowed for greater sensitivity to contextual effects.

\section{Conclusion}

In summary, we have shown that activation of early visual cortex is dependent on the relative perceived size of objects as opposed to their actual size. This has significant implications not only for perceptual science, but also for the treatment of mental health disorders. It has been shown that schizophrenia patients tend to demonstrate more veridical perception when viewing stimuli that normally creates visual illusions and that involves contextual integration (reviewed in Silverstein \& Keane, 2011), including those where size contrast (Silverstein et al., 2013) and depth perception (Keane, Silverstein, Wang, \& Papathomas, 2013) are involved. This reduced sensitivity to visual context is especially pronounced in acutely psychotic patients, but lessens with relapse and recovery as the illusion effects tend to normalize with symptom remission (Silverstein et al., 2013; Uhlhaas, Phillips, \& Silverstein, 2005). Because these behavioral impairments are linked to severity of disorganized (e.g., fragmented speech) and psychotic (e.g., hallucinations, delusions) symptoms respectively, it will be worthwhile to explore the extent to which reduced contextual effects in visual cortex serve as a biomarker of the connectivi- 
ty alterations that are thought to subserve these symptoms (Corlett, Frith, \& Fletcher, 2009; Corlett, Honey, Krystal, \& Fletcher, 2011; Phillips \& Silverstein, 2003; Uhlhaas, Phillips, Mitchell, \& Silverstein, 2006). Using the current paradigm could help reveal whether these size constancy abnormalities in schizophrenia had a functional basis in primary visual cortex. From there, it may be possible to examine if V1 volume or shape is related to activation level during size context processing, or if these differences result from other mechanisms known to alter receptive field size, such as attention (Fang et al., 2008) and perceptual load (de Haas, Schwarzkopf, Anderson, \& Rees, 2014).

\section{Acknowledgements}

This research was supported by the Biomedical Science Education Postdoctoral Training Program—NIH Grant No: 5K12GM093854, a Rutgers University grant supported RUBIC pilot work.

\section{Conflicts of Interest}

All authors declare that the research was conducted in the absence of any commercial or financial relationships that could be construed as a potential conflict of interest.

\section{References}

Angelucci, A., Levitt, J. B., Walton, E. J. S., Hupe, J.-M., Bullier, J., \& Lund, J. S. (2002). Circuits for Local and Global Signal Integration in Primary Visual Cortex. Journal of Neuroscience, 22, 8633-8646.

Arnold, D. H., Birt, A., \& Wallis, T. S. (2008). Perceived Size and Spatial Coding. Journal of Neuroscience, 28, $5954-5958$. http://dx.doi.org/10.1523/JNEUROSCI.0578-08.2008

Carlson, T. A., Ritchie, J. B., Kriegeskorte, N., Durvasula, S., \& Ma, J. (2014). Reaction Time for Object Categorization Is Predicted by Representational Distance. Journal of Cognitive Neuroscience, 26, 132-142. http://dx.doi.org/10.1162/jocn_a_00476

Corlett, P. R., Frith, C. D., \& Fletcher, P. C. (2009). From Drugs to Deprivation: A Bayesian Framework for Understanding Models of Psychosis. Psychopharmacology, 206, 515-530. http://dx.doi.org/10.1007/s00213-009-1561-0

Corlett, P. R., Honey, G. D., Krystal, J. H., \& Fletcher, P. C. (2011). Glutamatergic Model Psychoses: Prediction Error, Learning, and Inference. Neuropsychopharmacology, 36, 294-315. http://dx.doi.org/10.1038/npp.2010.163

Damaraju, E., Huang, Y. M., Barrett, L. F., \& Pessoa, L. (2009). Affective Learning Enhances Activity and Functional Connectivity in Early Visual Cortex. Neuropsychologia, 47, 2480-2487.

http://dx.doi.org/10.1016/j.neuropsychologia.2009.04.023

de Haas, B., Schwarzkopf, D. S., Anderson, E. J., \& Rees, G. (2014). Perceptual Load Affects Spatial Tuning of Neuronal Populations in Human Early Visual Cortex. Current Biology, 24, R66-R67. http://dx.doi.org/10.1016/j.cub.2013.11.061

Doherty, M. J., Campbell, N. M., Tsuji, H., \& Phillips, W. A. (2010). The Ebbinghaus Illusion Deceives Adults but Not Children. Developmental Science, 13, 714-721. http://dx.doi.org/10.1111/j.1467-7687.2009.00931.x

Doherty, M. J., Tsuji, H., \& Phillips, W. A. (2008). The Context Sensitivity of Visual Size Perception Varies across Cultures. Perception, 37, 1426-1433. http://dx.doi.org/10.1068/p5946

Fang, F., Boyaci, H., Kersten, D., \& Murray, S. O. (2008). Attention-Dependent Representation of a Size Illusion in Human V1. Current Biology, 18, 1707-1712. http://dx.doi.org/10.1016/j.cub.2008.09.025

Freeman, E., Sagi, D., \& Driver, J. (2001). Lateral Interactions between Targets and Flankers in Low-Level Vision Depend on Attention to the Flankers. Nature Neuroscience, 4, 1032-1036. http://dx.doi.org/10.1038/nn728

Friston, K., Rotshtein, P., Geng, J. J., Sterzer, P., \& Henson, R. N. (2006). A Critique of Functional Localisers. Neuroimage, 30, 1077-1087. http://dx.doi.org/10.1016/j.neuroimage.2005.08.012

Gilbert, C., Ito, M., Kapadia, M., \& Westheimer, G. (2000). Interactions between Attention, Context and Learning in Primary Visual Cortex. Vision Research, 40, 1217-1226. http://dx.doi.org/10.1016/S0042-6989(99)00234-5

Hu, K., Padmala, S., \& Pessoa, L. (2013). Interactions between Reward and Threat during Visual Processing. Neuropsychologia, 51, 1763-1772. http://dx.doi.org/10.1016/j.neuropsychologia.2013.05.025

Jenkinson, M., Bannister, P., Brady, M., \& Smith, S. (2002). Improved Optimization for the Robust and Accurate Linear Registration and Motion Correction of Brain Images. Neuroimage, 17, 825-841. http://dx.doi.org/10.1006/nimg.2002.1132

Kaas, J. H. (2000). Why Is Brain Size So Important: Design Problems and Solutions as Neocortex Gets Bigger or Smaller. Brain and Mind, 1, 7-23. http://dx.doi.org/10.1023/A:1010028405318 
Kanai, R., \& Rees, G. (2011). The Structural Basis of Inter-Individual Differences in Human Behaviour and Cognition. Nature Reviews Neuroscience, 12, 231-242. http://dx.doi.org/10.1038/nrn3000

Keane, B. P., Silverstein, S. M., Wang, Y., \& Papathomas, T. V. (2013). Reduced Depth Inversion Illusions in Schizophrenia Are State-Specific and Occur for Multiple Object Types and Viewing Conditions. Journal of Abnormal Psychology, 122, 506-512. http://dx.doi.org/10.1037/a0032110

Keil, A., Bradley, M. M., Ihssen, N., Heim, S., Vila, J., Guerra, P., \& Lang, P. J. (2010). Defensive Engagement and Perceptual Enhancement. Neuropsychologia, 48, 3580-3584. http://dx.doi.org/10.1016/j.neuropsychologia.2010.08.007

Kret, M. E., \& de Gelder, B. (2010). Social Context Influences Recognition of Bodily Expressions. Experimental Brain Research, 203, 169-180. http://dx.doi.org/10.1007/s00221-010-2220-8

Li, W., Piech, V., \& Gilbert, C. D. (2008). Learning to Link Visual Contours. Neuron, 57, 442-451. http://dx.doi.org/10.1016/j.neuron.2007.12.011

Murray, S. O., Boyaci, H., \& Kersten, D. (2006). The Representation of Perceived Angular Size in Human Primary Visual Cortex. Nature Neuroscience, 9, 429-434. http://dx.doi.org/10.1038/nn1641

Ni, A. M., Murray, S. O., \& Horwitz, G. D. (2014). Object-Centered Shifts of Receptive Field Positions in Monkey Primary Visual Cortex. Current Biology, 24, 1653-1658. http://dx.doi.org/10.1016/j.cub.2014.06.003

Open Science, C. (2015). PSYCHOLOGY. Estimating the Reproducibility of Psychological Science. Science, 349, Article ID: aac4716. http://dx.doi.org/10.1126/science.aac4716

Papathomas, T. V., \& Bono, L. M. (2004). Experiments with a Hollow Mask and a Reverspective: Top-Down Influences in the Inversion Effect for 3-D Stimuli. Perception, 33, 1129-1138. http://dx.doi.org/10.1068/p5086

Peirce, J. W. (2007). PsychoPy—Psychophysics Software in Python. Journal of Neuroscience Methods, 162, 8-13. http://dx.doi.org/10.1016/j.jneumeth.2006.11.017

Peirce, J. W. (2008). Generating Stimuli for Neuroscience Using PsychoPy. Front Neuroinform, 2, 10. http://dx.doi.org/10.3389/neuro.11.010.2008

Phillips, W. A., Clark, A., \& Silverstein, S. M. (2015). On the Functions, Mechanisms, and Malfunctions of Intracortical Contextual Modulation. Neuroscience and Biobehavioral Reviews, 52, 1-20. http://dx.doi.org/10.1016/j.neubiorev.2015.02.010

Phillips, W. A., \& Silverstein, S. M. (2003). Convergence of Biological and Psychological Perspectives on Cognitive Coordination in Schizophrenia. Behavioral and Brain Sciences, 26, 65-82; Discussion 82-137. http://dx.doi.org/10.1017/S0140525X03000025

Pooresmaeili, A., Arrighi, R., Biagi, L., \& Morrone, M. C. (2013). Blood Oxygen Level-Dependent Activation of the Primary Visual Cortex Predicts Size Adaptation Illusion. Journal of Neuroscience, 33, 15999-16008. http://dx.doi.org/10.1523/JNEUROSCI.1770-13.2013

Reddy, L., \& Kanwisher, N. (2006). Coding of Visual Objects in the Ventral Stream. Current Opinion in Neurobiology, 16, 408-414. http://dx.doi.org/10.1016/j.conb.2006.06.004

Ritchie, J. B., Tovar, D. A., \& Carlson, T. A. (2015). Emerging Object Representations in the Visual System Predict Reaction Times for Categorization. PLoS Computational Biology, 11, e1004316. http://dx.doi.org/10.1371/journal.pcbi.1004316

Schwarzkopf, D. S., \& Rees, G. (2013). Subjective Size Perception Depends on Central Visual Cortical Magnification in Human v1. PLoS ONE, 8, e60550. http://dx.doi.org/10.1371/journal.pone.0060550

Schwarzkopf, D. S., Song, C., \& Rees, G. (2011). The Surface Area of Human V1 Predicts the Subjective Experience of Object Size. Nature Neuroscience, 14, 28-30. http://dx.doi.org/10.1038/nn.2706

Shipley, W., Gruber, C., Martin, T., \& Klein, M. (2009). Shipley Institute of Living Scale-2. Los Angeles, CA: Western Psychological Services.

Silverstein, S. M., Hatashita-Wong, M., Schenkel, L. S., Wilkniss, S., Kovacs, I., Feher, A. et al. (2006). Reduced TopDown Influences in Contour Detection in Schizophrenia. Cognitive Neuropsychiatry, 11, 112-132.

http://dx.doi.org/10.1080/13546800444000209

Silverstein, S. M., \& Keane, B. P. (2009). Perceptual Organization in Schizophrenia: Plasticity and State-Related Change. Learning and Perception, 1, 229-261. http://dx.doi.org/10.1556/LP.1.2009.2.111

Silverstein, S. M., \& Keane, B. P. (2011). Perceptual Organization Impairment in Schizophrenia and Associated Brain Mechanisms: Review of Research from 2005 to 2010. Schizophrenia Bulletin, 37, 690-699.

http://dx.doi.org/10.1093/schbul/sbr052

Silverstein, S. M., Keane, B. P., Wang, Y., Mikkilineni, D., Paterno, D., Papathomas, T. V., \& Feigenson, K. (2013). Effects of Short-Term Inpatient Treatment on Sensitivity to a Size Contrast Illusion in First-Episode Psychosis and Multiple-Episode 
Schizophrenia. Frontiers in Psychology, 4, 1-11. http://dx.doi.org/10.3389/fpsyg.2013.00466

Silverstein, S. M., Knight, R. A., Schwarzkopf, S. B., West, L. L., Osborn, L. M., \& Kamin, D. (1996). Stimulus Configuration and Context Effects in Perceptual Organization in Schizophrenia. Journal of Abnormal Psychology, 105, 410-420. http://dx.doi.org/10.1037/0021-843X.105.3.410

Smith, S. M. (2002). Fast Robust Automated Brain Extraction. Human Brain Mapping, 17, 143-155. http://dx.doi.org/10.1002/hbm.10062

Song, C., Schwarzkopf, D. S., \& Rees, G. (2013). Variability in Visual Cortex Size Reflects Tradeoff between Local Orientation Sensitivity and Global Orientation Modulation. Nature Communications, 4, 2201. http://dx.doi.org/10.1038/ncomms3201

Tootell, R. B., Hadjikhani, N. K., Vanduffel, W., Liu, A. K., Mendola, J. D., Sereno, M. I., \& Dale, A. M. (1998). Functional Analysis of Primary Visual Cortex (V1) in Humans. Proceedings of the National Academy of Sciences of the United States of America, 95, 811-817. http://dx.doi.org/10.1073/pnas.95.3.811

Uhlhaas, P. J., Phillips, W. A., Mitchell, G., \& Silverstein, S. M. (2006). Perceptual Grouping in Disorganized Schizophrenia. Psychiatry Research, 145, 105-117. http://dx.doi.org/10.1016/j.psychres.2005.10.016

Uhlhaas, P. J., Phillips, W. A., \& Silverstein, S. M. (2005). The Course and Clinical Correlates of Dysfunctions in Visual Perceptual Organization in Schizophrenia during the Remission of Psychotic Symptoms. Schizophrenia Research, 75, 183-192. http://dx.doi.org/10.1016/j.schres.2004.11.005

Vetter, P., \& Newen, A. (2014). Varieties of Cognitive Penetration in Visual Perception. Consciousness and Cognition, 27, 62-75. http://dx.doi.org/10.1016/j.concog.2014.04.007

Wilson, H. R., \& Wilkinson, F. (2015). From Orientations to Objects: Configural Processing in the Ventral Stream. Journal of Vision, 15, 4. http://dx.doi.org/10.1167/15.7.4

Woolrich, M. W., Ripley, B. D., Brady, M., \& Smith, S. M. (2001). Temporal Autocorrelation in Univariate Linear Modeling of FMRI Data. Neuroimage, 14, 1370-1386. http://dx.doi.org/10.1006/nimg.2001.0931

Zhang, N. R., \& Von der Heydt, R. (2010). Analysis of the Context Integration Mechanisms Underlying Figure-Ground Organization in the Visual Cortex. Journal of Neuroscience, 30, 6482-6496. http://dx.doi.org/10.1523/JNEUROSCI.5168-09.2010

Zhu, M., \& Rozell, C. J. (2013). Visual Nonclassical Receptive Field Effects Emerge from Sparse Coding in a Dynamical System. PLoS Computational Biology, 9, e1003191. http://dx.doi.org/10.1371/journal.pcbi.1003191 